\title{
An Expectation Maximization Based Method for Subcellular Particle Tracking Using Multi-angle TIRF Microscopy*
}

\author{
Liang Liang, Hongying Shen, Pietro De Camilli, \\ Derek K. Toomre, and James S. Duncan \\ Yale University, New Haven, CT 06520, USA \\ liang.liang@yale.edu
}

\begin{abstract}
Multi-angle total internal reflection fluorescence microscopy (MA-TIRFM) is a new generation of TIRF microscopy to study cellular processes near dorsal cell membrane in 4 dimensions $(3 \mathrm{D}+\mathrm{t})$. To perform quantitative analysis using MA-TIRFM, it is necessary to track subcellular particles in these processes. In this paper, we propose a method based on a MAP framework for automatic particle tracking and apply it to track clathrin coated pits (CCPs). The expectation maximization (EM) algorithm is employed to solve the MAP problem. To provide the initial estimations for the EM algorithm, we develop a forward filter based on the most probable trajectory (MPT) filter. Multiple linear models are used to model particle dynamics. For CCP tracking, we use two linear models to describe constrained Brownian motion and fluorophore variation according to CCP properties. The tracking method is evaluated on synthetic data and results show that it has high accuracy. The result on real data confirmed by human expert cell biologists is also presented.
\end{abstract}

\section{Introduction}

With the assistance of live cell microscopy, especially TIRFM due to its unique illumination mechanism, biologists can investigate the underlying mechanisms of cellular processes taking place near the cell cortex, and understand some disease processes. The cell cortex is the cell's portal for intercellular communication and integrates signaling and cytoskeleton remodeling to control exo- and endocytosis. Here, we focus on clathrin mediated endocytosis (CME), which is an essential cellular process 1 that cells use for the selective internalization of surface molecules and of extracellular material. The study of CME has profound implications in neuroscience and virology. For instance, CME is the major route for synaptic vesicle recycling in neurons critical for synaptic transmission [1, and it is also one of the pathways through which viruses enter cells [2]. To study the process, the membrane associated protein complexes, namely clathrin coated pits (CCPs), are usually imaged by TIRFM. However conventional TIRFM can not provide

\footnotetext{
* This work was supported in part by the Keck Foundation and NIH grants (R37NS036251 and P30-DK45735 to P.D.C.).
} 
accurate information about z-positions and relative fluorophore amounts of individual particles. For MA-TIRFM [3, a set of images are obtained by quickly varying the incident angle, which can reveal the 3D information of the particles.

Some particle tracking methods for biological applications have been proposed in the literature 45677. A joint probabilistic data association (JPDA) filter based method [4 is proposed to track microtubule tips whose trajectories often cross over each other. An interacting multiple model (IMM) filter based method [5] is applied to track quantum dots with changing motion patterns. This method assumes that one motion pattern can be well described by one linear model, which is not necessarily true for our application. Recently, we proposed a 2D particle tracking method using TIRFM [6], which does better than the method in [7] for CCP tracking by incorporating information on the properties of CCPs. However, it does not consider the uncertainties from the feature detection stage, which may lead to relatively low estimation accuracy.

In this paper, we present an automatic tracking method based on MAPBayesian analysis to find the most probable trajectories of individual particles in $3 \mathrm{D}+\mathrm{t}$ using MA-TIRFM. We adopt the basic ideas of probabilistic data association and multiple model method [8], and assume that particle dynamics at each time can be described by a set of models with a certain probability distribution. In section 2, we present the tracking framework and describe some details. In section 3, we report the evaluation results on synthetic datasets with different SNRs, and also show the result on real data.

\section{Method}

\subsection{The Tracking Framework}

Let $\mathbf{I}_{t}$ be the MA-TIRFM image stack acquired at time $t$ (stack index), consisting of $2 \mathrm{D}$ images taken at different angles. Let $\mathbf{X}_{t}$ be the joint state of all particles at time $t$, assembled from each particle's state $X_{t}^{(i)}$. Let $\mathbf{D}_{t}$ be the observation/measurement set, and $D_{t}^{(j)}$ be the observation of a single particle. The goal is to find particle states that maximize the posterior probability:

$$
\left\{\widehat{\mathbf{X}}_{t}\right\}_{t=1}^{t_{\max }}=\arg \max _{\left\{\mathbf{X}_{t}\right\}_{t=1}^{t_{\max }}} \log p\left(\left\{\mathbf{X}_{t}\right\}_{t=1}^{t_{\max }} \mid\left\{\mathbf{I}_{t}\right\}_{t=1}^{t_{\max }}\right)
$$

Here, $\left\{\mathbf{X}_{t}\right\}_{t=1}^{t_{\max }}=\left\{\mathbf{X}_{1}, \ldots, \mathbf{X}_{t_{\max }}\right\}$. Since it is difficult to find the optimal solution directly, a practical way is to run detection and tracking iteratively:

$$
\begin{gathered}
\text { Detection : }\left\{\widehat{\mathbf{D}}_{\tau}\right\}_{\tau=1}^{t}=\arg \max _{\left\{\mathbf{D}_{\tau}\right\}_{\tau=1}^{t}} \log p\left(\left\{\mathbf{D}_{\tau}\right\}_{\tau=1}^{t} \mid\left\{\widehat{\mathbf{X}}_{\tau}, \mathbf{I}_{\tau}\right\}_{\tau=1}^{t}\right) \\
\text { Tracking }:\left\{\widehat{\mathbf{X}}_{\tau}\right\}_{\tau=1}^{t}=\arg \max _{\left\{\mathbf{X}_{\tau}\right\}_{\tau=1}^{t}} \log p\left(\left\{\mathbf{X}_{\tau}\right\}_{\tau=1}^{t} \mid\left\{\widehat{\mathbf{D}}_{\tau}\right\}_{\tau=1}^{t}\right)
\end{gathered}
$$

Detection is to find the most likely features of the particles given the image data and previously estimated states (if available). We have developed an algorithm 
for 3D particle feature estimation (3D positions and relative amount of fluorophores) using MA-TIRFM similar to the method in [3. Tracking is to find the most probable states/trajectories of the particles given the observation data.

To solve the MAP problem (Eq.(3)), we develop an expectation maximization (EM) based algorithm. We also develop a forward filter based on the MPT filter 8,9 and the idea of probabilistic data association [4 to provide initial estimations for the EM algorithm. The models of particle dynamics are described in section 2.2. The EM algorithm and the forward filter are described in section 2.3 and 2.4 respectively. The tracking flow is presented in section 2.5.

\subsection{The Particle Dynamics Models}

We assume particle dynamics/modes can be modeled using linear state space models [8] with a certain probability distribution at each time. $\pi(k, n)$ is the prior transition probability for each particle to switch from mode (model) $n$ to $k$ when the current mode (model) is $n$, and $\sum_{k} \pi(k, n)=1$. Each model $k$ has a posterior probability $\phi_{t}^{(i, k)}$ for each particle $i$, and $\sum_{k} \phi_{t}^{(i, k)}=1$. Each model is given as

$$
\begin{aligned}
& X_{t}^{(i)}=F_{t}^{(k)} X_{t-1}^{(i)}+U_{t}^{(i, k)}+W_{t}^{(k)} \\
& D_{t}^{(j)}=H X_{t}^{(i)}+V_{t}^{(j)}
\end{aligned}
$$

Here, $F_{t}^{(k)}$ is the state transition matrix. $U_{t}^{(i, k)}$ is the external input that we use to impose constraints. $W_{t}^{(k)}$ is the process noise with covariance matrix $Q_{t}^{(k)}$ learned from training data. $H$ is a constant observation matrix. $V_{t}^{(j)}$ is the observation noise with covariance matrix $R_{t}^{(j)}$ provided by the detection module. Each of these noise sources is assumed to be Gaussian and independent.

We define the state of each CCP $i$ at time $t$ as $X_{t}^{(i)}=\left[x_{t}, y_{t}, z_{t}, c_{t}, \dot{c}_{t}\right]^{\prime}$. $\left[x_{t}, y_{t}, z_{t}\right]$ is the position of the particle. $c_{t}$ is the relative amount of fluorophores in the particle, and $\dot{c}_{t}$ is the rate of change of fluorophores over time. We propose to use two linear state space models. For particle motion, the first model describes it as free Brownian motion because the motion is indeed random, and the second model describes it as confined motion because each CCP is linked to the plasma membrane through its neck [16. For fluorophore variation in each CCP, both models describe it as a linear process. The parameters are given by

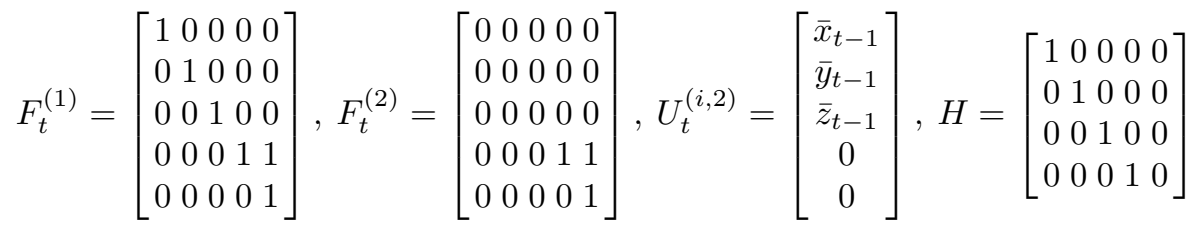

$U_{t}^{(i, 1)}$ is a zero vector for the first model, and $U_{t}^{(i, 2)}$ is the expected position of the particle determined by its neck joint. $\left[\bar{x}_{t}, \bar{y}_{t}, \bar{z}_{t}\right]$ is the time-average position, i.e., $\bar{x}_{t}=\frac{1}{t-t_{0}+1} \sum_{\tau=t_{0}}^{t} x_{\tau}$, and $t_{0}$ is the starting-time of the trajectory. Since each model reveals just half truth, we set the prior transition probabilities to be equal. The initial model probabilities are given as $\phi_{1}^{(i, 1)}=1$ and $\phi_{1}^{(i, 2)}=0$. 


\subsection{The EM Algorithm}

In the literature 8, some EM-based algorithms have already been proposed for general multiple target tracking, in which either the model probabilities or the data association probabilities are hidden variables. Here, we treat all of them as hidden variables, and combine deterministic annealing with the EM algorithm, and make some simplifications.

The lower bound $\mathcal{B}$ of the objective function (Eq.(3)) is constructed as:

$$
\begin{aligned}
\mathcal{B}\left(\left\{\mathbf{X}_{\tau}\right\}_{\tau=1}^{t} ;\left\{\widetilde{\mathbf{X}}_{\tau}\right\}_{\tau=1}^{t}\right)= & \mathrm{E}\left[\log p\left(\left\{\mathbf{D}_{\tau}, \mathbf{X}_{\tau}, \mathbf{A}_{\tau}, \mathbf{\Phi}_{\tau}\right\}_{\tau=1}^{t}\right) \mid\left\{\mathbf{D}_{\tau}, \widetilde{\mathbf{X}}_{\tau}\right\}_{\tau=1}^{t}\right] \\
& +\mathcal{H}\left(p\left(\left\{\mathbf{A}_{\tau}, \mathbf{\Phi}_{\tau}\right\}_{\tau=1}^{t} \mid\left\{\mathbf{D}_{\tau}, \widetilde{\mathbf{X}}_{\tau}\right\}_{\tau=1}^{t}\right)\right)
\end{aligned}
$$

Here, $\left\{\widetilde{\mathbf{X}}_{\tau}\right\}_{\tau=1}^{t}$ is the estimated states from the previous iteration. $\mathcal{H}(p)$ is the entropy of the probability distribution $p . \mathbf{A}_{\tau}$ is the binary matrix of data association. $\mathbf{A}_{\tau}(i, j)=1$ is the event that the observation $D_{\tau}^{(j)}$ is from the particle $i$ at time $\tau$, and $\mathbf{A}_{\tau}(i, 0)=1$ is the event that the particle $i$ disappears, and $\mathbf{A}_{\tau}(0, j)=1$ is the event that a new particle appears with observation $D_{\tau}^{(j)}$. Let $a_{\tau}^{(i, j)}$ be the data association probability, $a_{\tau}^{(i, j)}=p\left(\mathbf{A}_{\tau}(i, j)=1 \mid\left\{\mathbf{D}_{\tau}, \widetilde{\mathbf{X}}_{\tau}\right\}_{\tau=1}^{t}\right)$ with $\sum_{i>0} a_{\tau}^{(i, j)}=1$ and $\sum_{j>0} a_{\tau}^{(i, j)}=1$ assuming one-to-one correspondences. $\boldsymbol{\Phi}_{\tau}$ is the binary matrix of model assignment. $\boldsymbol{\Phi}_{\tau}(i, k)=1$ means the $k^{t h}$ model matches the dynamics of the particle $i$ at time $\tau$. The model probability $\phi_{\tau}^{(i, k)}$ is defined as $\phi_{\tau}^{(i, k)}=p\left(\boldsymbol{\Phi}_{\tau}(i, k)=1 \mid\left\{\mathbf{D}_{\tau}, \widetilde{\mathbf{X}}_{\tau}\right\}_{\tau=1}^{t}\right)$ with $\sum_{k} \phi_{\tau}^{(i, k)}=1$.

By using Eq.(4)-(6) and assuming that $\mathbf{A}_{\tau}$ and $\boldsymbol{\Phi}_{\tau}$ are statistically independent given the states and observation data, and ignoring constant terms, and combining deterministic annealing method, we obtain the expression of $\mathcal{B}$ :

$$
\begin{aligned}
\mathcal{B}= & -\frac{1}{2} \sum_{\tau=1}^{t} \sum_{i, j, k} a_{\tau}^{(i, j)} \phi_{\tau}^{(i, k)}\left(\left\|X_{\tau}^{(i)}-F_{\tau}^{(k)} X_{\tau-1}^{(i)}-U_{\tau}^{(i, k)}\right\|_{Q_{\tau}^{(k)}}^{2}+\log \left(\left|Q_{\tau}^{(k)}\right|\right)\right. \\
& \left.+\left\|D_{\tau}^{(j)}-H X_{\tau}^{(i)}\right\|_{R_{\tau}^{(j)}}^{2}+\log \left(\left|R_{\tau}^{(j)}\right|\right)\right)-T \sum_{\tau=1}^{t} \sum_{i, j} a_{\tau}^{(i, j)} \log \left(a_{\tau}^{(i, j)}\right) \\
& -\sum_{\tau=1}^{t} \sum_{i, k} \phi_{\tau}^{(i, k)} \log \left(\phi_{\tau}^{(i, k)}\right)+\sum_{\tau=1}^{t} \sum_{i, k} \phi_{\tau}^{(i, k)} \sum_{n} \phi_{\tau-1}^{(i, n)} \log \pi(k, n)
\end{aligned}
$$

Here, the vector norm is defined as $\|V\|_{L}^{2}=V^{\prime} L^{-1} V . T$ is the annealing temperature. $D_{\tau}^{(0)}$ is virtual observation specified for each particle similar to those in [6], which is commonly used to handle particle disappearing.

The exact formula for $a_{\tau}^{(i, j)}$ is very complicated for implementation, and therefore we make some approximations. By taking the derivative of $\mathcal{B}$ with respect to $a_{\tau}^{(i, j)}$ at $\left\{\mathbf{X}_{\tau}\right\}_{\tau=1}^{t}=\left\{\widetilde{\mathbf{X}}_{\tau}\right\}_{\tau=1}^{t}$, setting the result to zero, and making some modifications for robustness purpose, we obtain: 


$$
a_{\tau}^{(i, j)} \propto \exp \left[\frac{-1}{T} \sum_{k} \phi_{\tau}^{(i, k)}\left(\left\|H\left(\widetilde{X}_{\tau}^{(i)}-\widetilde{X}_{\tau \mid \tau-1}^{(i, k)}\right)\right\|_{\widetilde{Q}_{\tau}^{(k)}}^{2}+\left\|D_{\tau}^{(j)}-H \widetilde{X}_{\tau}^{(i)}\right\|_{R_{\tau}^{(j)}}^{2}\right)\right]
$$

Here, $\widetilde{X}_{\tau \mid \tau-1}^{(i, k)}=F_{\tau}^{(k)} \widetilde{X}_{\tau-1}^{(i)}+U_{\tau}^{(i, k)}$, and $\widetilde{Q}_{\tau}^{(k)}=H Q_{\tau}^{(k)} H^{\prime}$. The softassign algorithm [10] is applied to ensure that $\sum_{i>0} a_{\tau}^{(i, j)}=1$ and $\sum_{j>0} a_{\tau}^{(i, j)}=1$.

Similarly, we can obtain the update formula for $\phi_{\tau}^{(i, k)}$ :

$$
\begin{aligned}
\phi_{\tau}^{(i, k)} & \propto \exp \left[-\frac{1}{2}\left\|H\left(\widetilde{X}_{\tau}^{(i)}-\widetilde{X}_{\tau \mid \tau-1}^{(i, k)}\right)\right\|_{\widetilde{Q}_{\tau}^{(k)}}^{2}\right. \\
& \left.+\sum_{n} \phi_{\tau-1}^{(i, n)} \log \pi(k, n)+\sum_{n} \phi_{\tau+1}^{(i, n)} \log \pi(n, k)\right]
\end{aligned}
$$

Therefore, in the E-step, the probabilities of models and data associations are calculated using Eq.(8)-(9). In the M-step, the particle states that maximize $\mathcal{B}$ are estimated, which is achieved by taking the derivative of $\mathcal{B}$ with respect to the states of each particle, setting the result to zero, and solving a linear system of equations. In this step, the terms containing virtual observations are ignored. After each EM iteration, the annealing temperature is reduced. Eventually each $a_{\tau}^{(i, j)}$ converges to 0 or 1 , and one particle state has one observation.

\subsection{The Forward Filter}

We develop a forward filter based on the most probable trajectory (MPT) filter 89 that was proposed for tracking a single target with multiple models. The forward filter handles the data association problem in a manner consistent with the EM algorithm, and provides state estimation and prediction given the observation data. Following the similar derivation process and notations in [9], we obtain the prediction and update formulas for each particle's state:

$$
\begin{aligned}
X_{t+1 \mid t}^{(i)} & =K_{t+1}^{-1}\left[\overline{Q U_{t}}+\overline{Q F_{t}} S_{t}\left(-\overline{F^{\prime} Q U_{t}}+\overline{H^{\prime} R D_{t}}+K_{t} X_{t \mid t-1}^{(i)}\right)\right] \\
X_{t \mid t}^{(i)} & =\left(\overline{F_{t}}\right)^{-1}\left(X_{t+1 \mid t}^{(i)}-\overline{U_{t}}\right) \\
S_{t} & =\left(\overline{F^{\prime} Q F_{t}}+\overline{H^{\prime} R H_{t}}+K_{t}\right)^{-1} \\
K_{t+1} & =\overline{Q_{t}}-\overline{Q F_{t}} S_{t} \overline{F^{\prime} Q_{t}}
\end{aligned}
$$

Here, we define $\overline{G_{t}}=\sum_{k} \phi_{t}^{(i, k)} G_{t}^{(i, k)}$ and $\overline{G Q P_{t}}=\sum_{k} \phi_{t}^{(i, k)} G_{t}^{(i, k)}\left(Q_{t}^{(k)}\right)^{-1} P_{t}^{(i, k)}$ for any matrix $G$ and $P$, e.g., $\overline{F^{\prime} Q U_{t}}=\sum_{k} \phi_{t}^{(i, k)}\left(F_{t}^{(k)}\right)^{\prime}\left(Q_{t}^{(k)}\right)^{-1} U_{t}^{(i, k)}$, and $\overline{H^{\prime} R H_{t}}=\sum_{j>0} a_{t}^{(i, j)} H^{\prime}\left(R_{t}^{(j)}\right)^{-1} H$, and $\overline{H^{\prime} R D_{t}}=\sum_{j>0} a_{t}^{(i, j)} H^{\prime}\left(R_{t}^{(j)}\right)^{-1} D_{t}^{(j)}$. 
We define the data association probabilities and model probabilities as:

$$
\begin{aligned}
a_{t}^{(i, j)} & \propto \exp \left[-\sum_{k} \phi_{t \mid t-1}^{(i, k)}\left\|D_{t}^{(j)}-H X_{t \mid t-1}^{(i)}\right\|_{\tilde{Q}_{t}^{(k)}}^{2}\right] \\
\phi_{t}^{(i, k)} & \propto \phi_{t \mid t-1}^{(i, k)} \exp \left[-\frac{1}{2} \sum_{j} a_{t}^{(i, j)}\left\|D_{t}^{(j)}-H\left(F_{t}^{(k)} X_{t-1 \mid t-1}^{(i)}+U_{t}^{(i, k)}\right)\right\|_{\tilde{Q}_{t}^{(k)}}^{2}\right] \\
\phi_{t \mid t-1}^{(i, k)} & =\sum_{n} \pi(k, n) \phi_{t-1}^{(i, n)}
\end{aligned}
$$

Here, $\sum_{k} \phi_{t}^{(i, k)}=1, \sum_{i>0} a_{t}^{(i, j)}=1$ and $\sum_{j>0} a_{t}^{(i, j)}=1$.

\subsection{The Tracking Flow}

After the detection module runs on the image stacks, the tracking module starts with the forward filter to obtain state estimations in the time window $[t, t+w]$. Then, the EM algorithm runs in the time window to update the relevant estimations. Given the updated states as prior information, in the time window the detection module runs again to refine the detection results and the EM algorithm runs again to refine state estimations. Then, the forward filter is reinitialized at time $t+w$, and the window slides, and the process is repeated. During the track-

ing process, a track $i$ is terminated when $a_{t}^{(i, 0)}$ is greater than a threshold (0.9 in experiments), and new tracks are initialized if the number of observations is greater than the number of existing particles.

\section{Experimental Results}

\subsection{Evaluation on Synthetic Data}

The proposed method is evaluated on synthetic MA-TIRFM image datasets. Each dataset contains 200 image stacks, and each image stack consists of four images acquired at penetration depth $100 \mathrm{~nm}, 200 \mathrm{~nm}, 300 \mathrm{~nm}$, and $400 \mathrm{~nm}$. Gaussian distributions are fitted to the histogram of particle displacements and to the histogram of the deviations from the mean positions of individual particles, and therefore trajectories can be created by sampling from the distributions. Three major types of noises are considered in the simulation process, the thermal noise of laser, the Poisson (shot) noise of input photon, and the excess noise generated in the EMCCD. The SNR of each dataset can be tuned by varying the ratio of the average fluorophore amount of individual CCPs and the background fluorophore concentration. We also test an alternative method which is essentially the same as the proposed method except that it only uses the first model described in section 2.2 .

The results are shown in Fig. 1(a)-(h). Tracking accuracies (defined in [6]) under different SNRs are shown in Fig.1(a). The proposed method is consistently better. The alternative method produces some long but wrong trajectories 


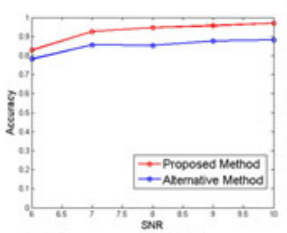

(a) Accuracy v.s. SNR

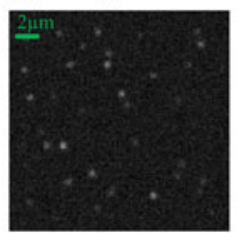

(e) Synthetic image $(\mathrm{d}=100 \mathrm{~nm})$

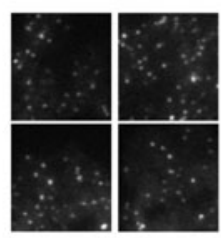

(i) Real images

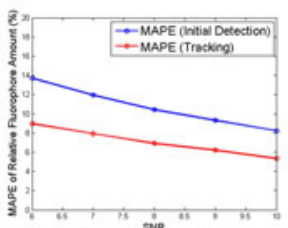

(b) MAPE of Fluorophores

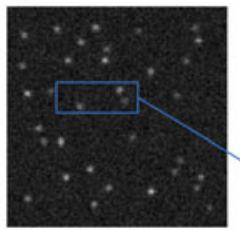

(f) Synthetic image (d=400nm)

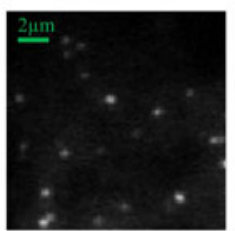

(j) Image sample (d=64nm)

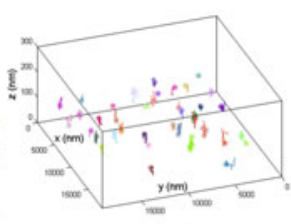

(c) Samples (proposed method)

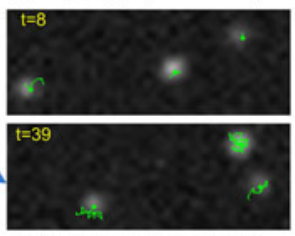

(g) Samples (proposed method)

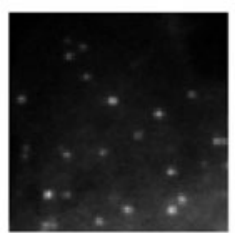

(k) Image sample $(\mathrm{d}=\infty)$

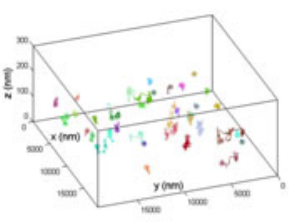

(d) Samples (alternative method)

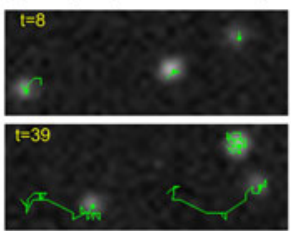

(h) Samples (alternative method)

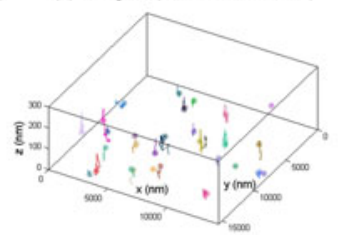

(1) Real trajectory samples

Fig. 1. (a) Accuracy-SNR curves. (b) MAPE of the estimated relative amount of fluorophores. (c)\&(d) Trajectory samples in 3D. (e)\&(f) Synthetic images at $d=100 \mathrm{~nm}$ and $d=400 \mathrm{~nm}$. (g) \&(h) Trajectory samples in 2D. (i) Image samples of real datasets. (j) \&(k) Real images acquired at $d=64 n m$ and $d=\infty$. (l) Real trajectory samples obtained by using the proposed method.

(Fig.1(h)), which happens most likely when new CCPs appear near the positions where matured CCPs disappear. The result is consistent with what is reported in [6]. In Fig.1(b), we show the mean absolute percentage error (MAPE) of the estimated relative amount of fluorophores. The MAPE calculated after tracking (using the proposed method) is smaller than the MAPE calculated after initial detection. Since the uncertainties from the detection stage are considered in the tracking framework, the method can improve feature estimation accuracy.

\subsection{Evaluation on Real Data}

Mouse fibroblast cells were electroporated with clathrin light chain GFP construct using the Amaxa Nucleofector method, and were plated at subconfluent densities into 35mm glass bottom dishes, and allowed to grow for 12 to 48 hours. Images were acquired by Nikon Ti-E Eclipse inverted microscope with Apo TIRF $100 \times$ oil objective lens (NA 1.49) and Andor iXon 897 EMCCD camera. For the initial evaluation, two angles were used. The first one corresponds to penetration depth $d=64 \mathrm{~nm}$, to observe the particles near the glass slide. The second one is greater than the critical angle, to observe particles in the deeper range. Although total internal reflection will not occur with the second angle, the axial profile of the light field can still be modeled as an exponential function $f(z)=I_{0} e^{-z / d}=I_{0}$ 
with $d=\infty$. Mathematically, it does not make any difference to the method. Regions of interest are selected from image datasets, and the tracking results are visually inspected and confirmed by human expert cell biologists. Samples of image regions and trajectories are shown in Fig. 1 (i)-(l).

\section{Conclusion}

We have proposed an automatic particle tracking method based on a MAP framework and applied it to track clathrin coated pits (CCPs) in $3 \mathrm{D}+\mathrm{t}$ using MA-TIRFM. We use a set of linear models to model particle dynamics, and develop the EM-based algorithm and the forward filter to solve the MAP problem. We evaluate the method using simulation with different SNRs, and also test it on real data. The results show the method is promising for the analysis of CCPs. In the near future, we'll optimize MA-TIRFM imaging parameters and obtain images with four penetration depths and two fluorescent channels.

\section{References}

1. Slepnev, V.I., De Camilli, P.: Accessory factors in clathrin-dependent synaptic vesicle endocytosis. Nature Reviews Neuroscience 1, 161-172 (2000)

2. Brandenburg, B., Zhuang, X.: Virus trafficking - learning from single-virus tracking. Nature Reviews Microbiology 5, 197-208 (2007)

3. Yang, Q., Karpikov, A., Toomre, D., Duncan, J.S.: 3D reconstruction of microtubules from multi-angle total internal reflection fluorescence microscopy using Bayesian framework. IEEE Trans. on Image Processing (2011) (in press)

4. Smal, I., Niessen, W., Meijering, E.: A new detection scheme for multiple object tracking in fluorescence microscopy by joint probabilistic data association filtering. In: IEEE Int. Symposium on Biomedical Imaging: From Nano to Macro, pp. 264267 (2008)

5. Genovesio, A., Liedl, T., Emiliani, V., Parak, W.J., Coppey-Moisan, M., OlivoMarin, J.-C.: Multiple particle tracking in 3-D+t microscopy: method and application to the tracking of endocytosed quantum dots. IEEE Trans. on Image Processing 15(5), 1062-1070 (2006)

6. Liang, L., Shen, H., De Camilli, P., Duncan, J.S.: Tracking clathrin coated pits with a multiple hypothesis based method. In: Jiang, T., Navab, N., Pluim, J.P.W., Viergever, M.A. (eds.) MICCAI 2010. LNCS, vol. 6362, pp. 315-322. Springer, Heidelberg (2010)

7. Jaqaman, K., Loerke, D., Mettlen, M., Kuwata, H., Grinstein, S., Schmid, S.L.L., Danuser, G.: Robust single-particle tracking in live-cell time-lapse sequences. Nature methods 5, 695-702 (2008)

8. Li, X.R., Jilkov, V.P.: Survey of maneuvering target tracking. Part I. Dynamic models. IEEE Trans. on Aerospace and Electronic Systems 39(4), 1333-1364 (2003)

9. Zhang, Q.: Optimal filtering of discrete-time hybrid systems. Journal of Optimization Theory and Applications 100(1), 123-144 (1999)

10. Rangarajan, A., Chui, H., Bookstein, F.L.: The softassign procrustes matching algorithm. In: Duncan, J.S., Gindi, G. (eds.) IPMI 1997. LNCS, vol. 1230, pp. 29-42. Springer, Heidelberg (1997) 